\title{
The extremely red host galaxy of GRB 080207
}

\author{
Leslie Hunt \\ INAF-Osservatorio Astrofisico di Arcetri, Largo E. Fermi 5, I-50125 Firenze, Italy \\ hunt@arcetri.astro.it \\ Eliana Palazzi \\ INAF-IASF Bologna, Via Gobetti 101, I-40129 Bologna, Italy
}

Andrea Rossi

Thüringer Landessternwarte Tautenburg, Sternwarte 5, D-07778 Tautenburg, Germany

Sandra Savaglio

Max-Planck-Institut für Extraterrestrische Physik, Giessenbachstraße, D-85748 Garching bei München, Germany

Giovanni Cresci

INAF-Osservatorio Astrofisico di Arcetri, Largo E. Fermi 5, I-50125 Firenze, Italy

Sylvio Klose

Thüringer Landessternwarte Tautenburg, Sternwarte 5, D-07778 Tautenburg, Germany

\begin{abstract}
Michał Michałowski
Scottish Universities Physics Alliance, Institute for Astronomy, University of Edinburgh, Royal Observatory, Edinburgh, EH9 $3 H J, U K$

and
\end{abstract}

\section{Elena Pian}

INAF, Trieste Astronomical Observatory, via G.B. Tiepolo 11, 34143 Trieste, Italy; Scuola Normale Superiore, Piazza dei Cavalieri 7, 56126 Pisa, Italy

\begin{abstract}
We present optical, near-infrared, and Spitzer IRAC and MIPS observations of the host galaxy of the dark gamma-ray burst GRB 080207 . The host is faint, with extremely red optical-infrared colors $(R-K=6.3,24 \mu \mathrm{m} / R$-band flux $\sim 1000)$ making it an extremely red object (ERO) and a dust-obscured galaxy (DOG). The spectral energy distribution (SED) shows the clear signature of the $1.6 \mu \mathrm{m}$ photometric "bump", typical of evolved stellar populations. We use this bump to establish the photometric redshift $z_{\text {phot }}$ as $2.2_{-0.3}^{+0.2}$, using a vast library of SED templates, including M 82. The star-formation rate (SFR) inferred from the SED fitting is $\sim 119 M_{\odot} \mathrm{yr}^{-1}$, the stellar mass $3 \times 10^{11} M_{\odot}$, and $A_{V}$ extinction from 1-2 mag. The ERO and DOG nature of the host galaxy of the dark GRB 080207 may be emblematic of a distinct class of dark GRB hosts, with high SFRs, evolved and metal-rich stellar populations, and significant dust extinction within the host galaxy.

Subject headings: galaxies: high-redshift — galaxies: ISM — (ISM:) dust, extinction
\end{abstract}




\section{Introduction}

Gamma-Ray Bursts (GRBs) without an optical counterpart of their X-ray afterglow are usually called "dark bursts"; a more precise definition based on their X-ray to optical spectral energy distribution (SED) has been proposed (Jakobsson et al. 2004; Rol et al. 2005; van der Horst et al. 2009). It is clear now that in most cases extinction by dust in their host galaxies makes these afterglows optically dim (Greiner et al. 2011), while cosmological Lyman drop out (high redshift) or intrinsic faintness are the exception rather than the rule Cenko et al. 2009; Perley et al. 2009; Rossi et al. 2011).

The long-duration GRB 080207 is one of these truly dark GRBs. It was detected by Swift/BAT on 2008 February 7 at 21:30:21 UT. Swift/XRT began observing the field $124 \mathrm{~s}$ after the BAT trigger and found a bright X-ray afterglow, with a positional uncertainty radius of 1".4 (Racusin et al. 2008a). No optical/nearinfrared afterglow was detected for GRB 080207, despite heroic efforts by several ground-based observatories. The deepest limit relative to the fading X-ray afterglow was achieved by the Zeiss-600 telescope at Mt.Terskol observatory which did not detect the afterglow down to $R_{\mathrm{AB}}>20.5$ at $1.69 \mathrm{hr}$ after the burst (Andreev et al. 2008). Racusin et al. (2008b c) report that the XRT light curve between $1.30 \mathrm{hr}$ and $4.72 \mathrm{hr}$ after the GRB start time declines monotonically with a power-law of index $\alpha_{X}=1.85 \pm 0.10$. Therefore, we have assumed that the optical light curve in the same time interval is also decreasing monotonically, so that its behavior is sufficiently regular that the criterion of Jakobsson et al. (2004) to define GRB "darkness" based on the comparison of the X-ray and optical flux levels at $11 \mathrm{hr}$ - can be applied to the optical upper limit $R_{\mathrm{AB}}>20.5$ measured at $1.69 \mathrm{hr}$ after the trigger. The X-ray flux at $1.69 \mathrm{hr}$ is $\sim 0.35 \mathrm{c} / \mathrm{s}\left(\sim 3.1 \times 10^{-11}\right.$ $\mathrm{erg} \mathrm{s}^{-1} \mathrm{~cm}^{2}$ ), which, assuming the spectral index fitted by Racusin et al. to the average XRT spectrum in the above time interval, $\beta_{X}=1.4 \pm 0.2$, corresponds to a $1 \mathrm{keV}$ flux of $4.2 \mu \mathrm{Jy}$. Together with the simultaneous optical upper limit, this yields an optical-to-Xray index of $\beta_{O X}<0.27$, which leads to a dark GRB classification, according to Jakobsson et al. (2004) and van der Horst et al. (2009).

In the vicinity of the XRT error circle, Rossi et al. (2011) found two very faint host candidates, one better visible in VIMOS/ $R$-band and the second brighter in $K$. However, the Chandra satellite observed the field of GRB 080207 nine days after the burst detection by Swift (Racusin et al. 2008a), and was able to localize the GRB X-ray afterglow with very high accuracy of 0.67 (Evans et al. 2010): $\alpha=13: 50: 02.97$, $\delta=07: 30: 07.8$ (J2000). This position coincides with one of the candidates found by Rossi et al. (2011) (source "B"): a very faint $\left(R_{\mathrm{AB}} \sim 26.5 \mathrm{mag}\right)$, very red $\left[R-K \gtrsim 6,(R-K)_{\mathrm{AB}} \sim 4.7\right]$ galaxy. The other candidate is bluer (dubbed "A" by Rossi et al. 2011), with $(R-K)_{\mathrm{AB}} \sim 2.1$, and located just outside the $90 \%$ XRT error circle, $\sim 2$ !' 3 northeast of the Chandra position. Hence we associate the first source (B) with GRB 080207.

In this Letter, we present proprietary and archival optical and near-infrared (NIR) observations, combined with archival Spitzer IRAC and MIPS data of the host of GRB 080207. We estimate the photometric redshift $z_{\text {phot }}$ of the host by fitting the observed SED with a vast library of GRASIL models (Silva et al. 1998; Iglesias-Páramo et al. 2007; Michałowski et al. 2008, 2010). Despite its optical faintness, because of the clear photospheric NIR bump at rest-frame $\lambda=1.6 \mu \mathrm{m}$, we are able to estimate the redshift of the host of GRB 080207 with a precision of \pm 0.3 . Section 2 describes the data reduction and the methods used to derive the photometry. The SED models are presented in $\S 3$, and we discuss the results and their implications in $\S$. Throughout the paper, we assume a $\Omega_{m}=0.3, \Omega_{\Lambda}=0.7$ cosmology, with Hubble constant $H_{0}=70 \mathrm{~km} \mathrm{~s}^{-1} \mathrm{Mpc}^{-1}$.

\section{Data and photometry}

In the course of an analysis of GRB host galaxy SEDs (Hunt et al., in preparation), we culled the Spitzer archive for observations of GRB 080207. It was observed by A. Levan (PID 50562) more than one year after the burst in all four IRAC channels (Fazio et al. 2004) and about five months after the burst at MIPS $24 \mu \mathrm{m}$ (Rieke et al. 2004). $R$ - and $K$ band data were taken from Rossi et al. (2011). $B$ - and $R$-band images were acquired at the Large Binocular Telescope (LBT) in the course of our approved observing program; $i^{\prime}$ and $z^{\prime}$ images were taken from the Gemini archive, and an $H$-band image from the HST/NICMOS archive. Finally, we retrieved the ESO/SINFONI NIR integral field spectral data from the ESO archive, which provided a broad-band $J$ continuum point. Altogether we analyzed 12 photometric data points, from $0.4 \mu \mathrm{m}$ to $24 \mu \mathrm{m}$, in order to establish 
the photometric redshift and the general properties of the host of GRB 080207 . The magnitudes given below are corrected for foreground Galactic extinction assuming $E(B-V)=0.023 \mathrm{mag}$ (Schlegel et al. 1998) and a ratio of total-to-selective extinction of $R_{V}=3.1$.

\subsection{Optical and near-infrared observations}

We observed the field 2 years after the burst with LBT/LBC in the $B$ and $R$ bands, and with VLT/VIMOS in $R$ and VLT/ISAAC in $K_{s}$ bands. Optical and NIR data were reduced using standard IRAF task 1 , and analyzed through aperture photometry. We used an aperture size twice the Full Width Half Maximum (FWHM) of the stellar PSF. Optical VLT and LBT images were calibrated using Landolt stars in the field PG1047+3. ISAAC fields were calibrated using 2MASS field stars.

Within the Swift/XRT error circle of 1'.4, and coincident with afterglow localized by the Chandra satellite, an extended object $\left(1{ }^{\prime \prime} 6 \times 0.0^{\prime} 9\right)$ is visible in our LBC, VIMOS $R$-band, and ISAAC $K_{s}$-band images; it is thus identified as the optical counterpart of the GRB host galaxy (Rossi et al. 2011). The galaxy is marginally detected in both LBC and VIMOS $R$ band with $R_{\mathrm{AB}}=26.43 \pm 0.37$, but is clearly visible in our ISAAC images (see Fig. 1) $K_{s, \mathrm{AB}}=21.77 \pm 0.14$ ). The object is absent in our $B$-band LBT image, which is however compromised by worse seeing.

The host galaxy is also clearly visible in an archival Gemini/GMOS $z^{\prime}$ image obtained 6 months after the burst during very good sky conditions, but not in the $i^{\prime}$ image taken immediately after the GRB. We obtain $i_{\mathrm{AB}}^{\prime}>24.6$ and $z_{\mathrm{AB}}^{\prime}=25.02 \pm 0.02$.

We reduced archival $J$ (30 min exposure) and $H+K$ (45 min) band SINFONI integral field spectra around the host galaxy, following all the typical reduction steps applied to near-IR spectra. After background subtraction, the data were flat-fielded, corrected for dead/hot pixels, wavelength and flux calibrated using telluric stars. The resulting datacubes were collapsed to obtain broadband continuum images; although the final $\mathrm{S} / \mathrm{N}$ in the $H+K$ band was too low, the derived $J$ band image was analyzed using standard aperture photometry to extract the flux. The spectra themselves were of insufficient sensitivity for reliable detection of

${ }^{1}$ IRAF is distributed by the National Optical Astronomy Observatory, which is operated by the Association of Universities for Research in Astronomy (AURA) under cooperative agreement with the National Science Foundation.
[O II], [O III], or $\mathrm{H} \alpha$ emission lines (see $\$ 4$ ).

We reduced the HST/NICMOS F160W (NIC3) image starting with the standard flat-fielded calibrated frames after correcting for the pedestal effect. These frames were then "drizzled" onto the final mosaic (Fruchter \& Hook 2002), using a pixel filling factor of 0.65 , and maintaining the original pixel size of 0.2 . The host galaxy of GRB 080207 is clearly visible in this image also, and using the same methods as described above, we obtain a flux of $2.9 \pm 0.7 \mu \mathrm{Jy}$ (see Table 1).

\subsection{Spitzer IRAC and MIPS observations}

For both IRAC and MIPS observations, we started the data reduction with the Basic Calibrated Data (BCD) and the corresponding masks (DCE masks), furnished by the Spitzer Science Center (SSC) pipeline. The image mosaicing and source extraction package (MOPEX, Makovoz \& Marleau 2005) was used to co-add BCD frames for each source, and mask out bad pixels. For MIPS-24, we discarded the first two frames of each observation sequence, as advocated by the MIPS Instrument Handbook. Also for MIPS, we incorporated the sigma-weighting algorithm since it gave less noisy MIPS mosaics than without. The frames were corrected for geometrical distortion and projected onto a fiducial coordinate system with pixel sizes of 1'.20, roughly equivalent to the original IRAC pixels. The quality of our IRAC and MIPS maps is substantially better than that provided by the Spitzer archive.

Figure 1 shows the ISAAC $K$-band image taken from Rossi et al. (2011), together with the IRAC $3.6 \mu \mathrm{m}$ and MIPS $24 \mu \mathrm{m}$ images presented here. The Chandra afterglow position is marked with an $\times$. The brightest IRAC pixels correspond to the Chandra position, and to source "B" found by Rossi et al. (2011).

In all IRAC and MIPS Spitzer images we detected only one object within the Chandra error box which we identify as the GRB 080207 host galaxy. The object is not spatially resolved in the Spitzer images. We estimated the flux density in all filters using a small circular aperture with a radius of 2 pixels in IRAC and 3 pixels in MIPS; these measurements were performed using the phot task in IRAF. Aperture corrections have been applied to account for the extended size of the PSF relative to the small apertures.

Due to the possible contamination from nearby objects (see Fig. 1), we checked the resulting fluxes 


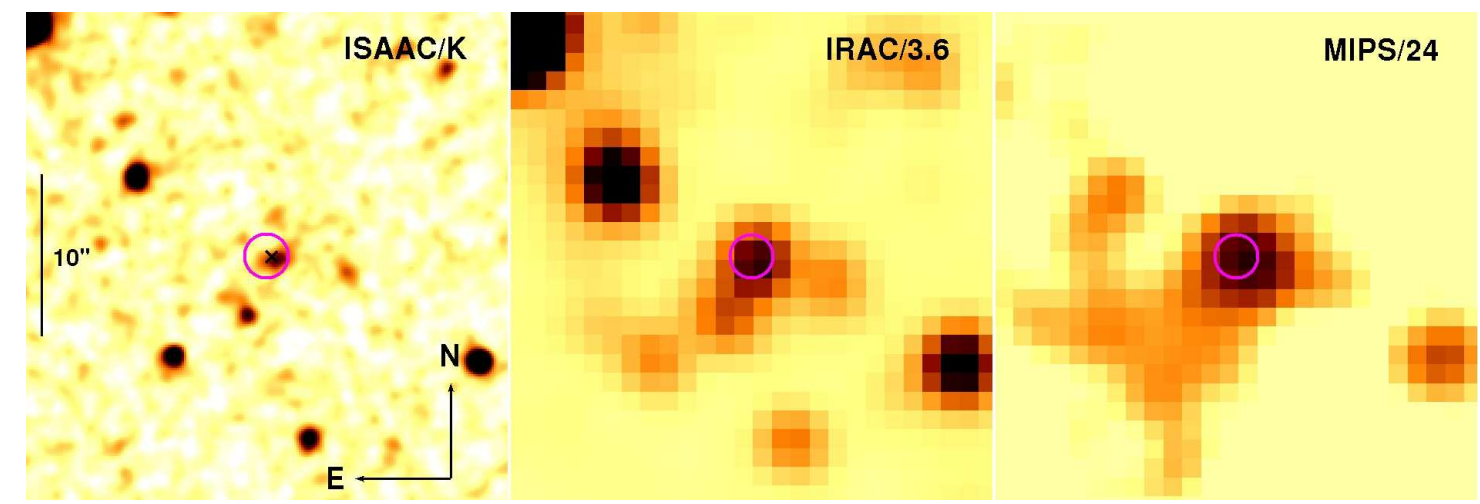

Fig. 1.- From left to right: the host galaxy of GRB 080207 in $K$ band (Rossi et al. 2011), in IRAC/3.6 $\mu$ m, and MIPS $/ 24 \mu \mathrm{m}$. North is up, East is left; the images are $30^{\prime \prime}$ on a side. The XRT error circle is shown, and the Chandra position is marked with $\times$.

by using PSF fitting with the DAOPHOT software (Stetson 1987) to perform the source extraction and photometry. The results from the two methods are perfectly consistent. Table 1 reports the fluxes we have derived and use in the SED fitting described below.

\section{Modeling the SED}

With $(R-K)_{\mathrm{AB}} \sim 4.7\left[(R-K)_{\text {Vega }} \sim 6.3\right]$, the host galaxy is very red, among the reddest host galaxies of a LGRB ever observed. Such red $R-K$ colors classify the host galaxy of GRB 080207 as an extremelyred object (ERO: Elston et al. 1988). The $R-24 \mu \mathrm{m}$ color is also sufficiently red $(24 \mu \mathrm{m} / R$ flux ratio $\sim 994)$ to classify it as a dust-obscured galaxy (DOG: $24 \mu \mathrm{m} / R$ flux ratio $\gtrsim 1000$, Dey et al. 2008).

Given the extremely dim optical fluxes, the strongest constraint on the SED we present here is the spectral "bump" at rest-frame $1.6 \mu \mathrm{m}$ (see Fig. 2). This bump is due to the minimum in the opacity in the $\mathrm{H}^{-}$ion found in the photospheres of cool stars, and found in virtually all stellar populations older than $\sim 100 \mathrm{Myr}$; it provides a conspicuous signature with which to constrain photometric redshifts Simpson \& Eisenhardt 1999; Sawicki 2002). The host of GRB 080207 is one of the few GRB hosts to show this feature (another one is ESO 184-G82, the host of GRB 980425, Michałowski et al. 2009). Because of its extremely red colors, the host of GRB 080207, is either a passive early-type galaxy at redshift $1<z<2$, or a dusty starburst at higher redshift. Because LGRBs occur in star-forming galaxies (e.g., Le Floc'h et al. 2003; Savaglio et al. 2009), we concentrated on the second alternative, and considered a library of 35000 GRASIL star-forming galaxy templates, including M 82 and Arp 220 and other GRB host templates (Silva et al. 1998; Iglesias-Páramo et al. 2007; Michałowski et al. 2008, 2010).

SED fitting in $v L_{v}$ space was performed by renormalizing the templates, to minimize $\chi^{2}$ over the observed $\mathrm{SED}^{2}$. This was done by redshifting the models over a range of redshifts from 0 to 6 , in increments of 0.1 . Lyman forest attenuation was treated according to Madau (1995). We used the fitting method described in Iglesias-Páramo et al. (2007); Michałowski et al. (2008, 2010) and one developed independently by our group; the two approaches give virtually identical results. The best fit was obtained for a starburst template, with roughly the same parameters as M 82, at a redshift $z=2.2$ (mean residuals $0.1 \mu \mathrm{Jy}$, reduced $\chi_{v}^{2}=0.3$ ). The best-fitting SED is shown in Fig. 2, and the trends with redshift of $\chi_{v}^{2}$ for representative GRASIL templates are shown in Fig. 3. After a statistical analysis of the $\chi^{2}$ distributions, we conclude that the best estimate of the photometric redshift for the host of GRB 080207 is $z=2.2_{-0.3}^{+0.2}$ (errors are $1 \sigma$, and were calculated including the choice of template as a free parameter).

The most salient feature of our SED fit is its ability to well fit the MIPS point, the $B$ and $i^{\prime}$ upper limits, and the $R, z^{\prime}$, and $J$-band fluxes. Even though the $24 \mu \mathrm{m}$ data point is not included in our fitting procedure, it is

\footnotetext{
${ }^{2}$ In order to better weight the NIR bump in the fits, we excluded both the $8 \mu \mathrm{m}$ IRAC point and the $24 \mu \mathrm{m}$ MIPS point in the fits; the fits were more stable with this exclusion.
} 
TABLE 1

Photometry of the host galaxy of GRB 080207

\begin{tabular}{ccccc}
\hline \hline Filter & $\begin{array}{c}(\lambda) \\
\mu \mathrm{m}\end{array}$ & $\begin{array}{c}\text { Flux }^{\mathrm{a}} \\
(\mu \mathrm{Jy})\end{array}$ & $\begin{array}{c}\text { Uncertainty } \\
\mu \mathrm{Jy}\end{array}$ & $\begin{array}{c}\text { Telescope/ } \\
\text { Instrument }\end{array}$ \\
\hline$B$ & 0.44 & $<0.15^{\mathrm{b}}$ & & LBT/LBC \\
$R$ & 0.66 & 0.093 & 0.026 & VLT/VIMOS \\
$i^{\prime}$ & 0.78 & $<0.32^{\mathrm{b}}$ & & Gemini/GMOS \\
$z^{\prime}$ & 0.925 & 0.35 & 0.06 & Gemini/GMOS \\
$J$ & 1.2 & 1.6 & 0.3 & VLT/SINFONI \\
$H$ & 1.60 & 2.9 & 0.7 & HST/NICMOS \\
$K_{s}$ & 2.2 & 7.3 & 1.0 & VLT/ISAAC \\
& 3.550 & 14.40 & 0.31 & Spitzer/IRAC \\
& 4.493 & 15.51 & 0.44 & Spitzer/IRAC \\
& 5.731 & 18.53 & 1.58 & Spitzer/IRAC \\
& 7.872 & 12.52 & 1.76 & Spitzer/IRAC \\
& 23.680 & 92.43 & 6.50 & Spitzer/MIPS \\
\hline
\end{tabular}

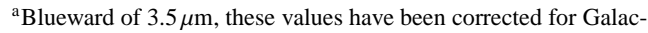
tic extinction as described in the text.

${ }^{\mathrm{b}} 3 \sigma$ upper limits.

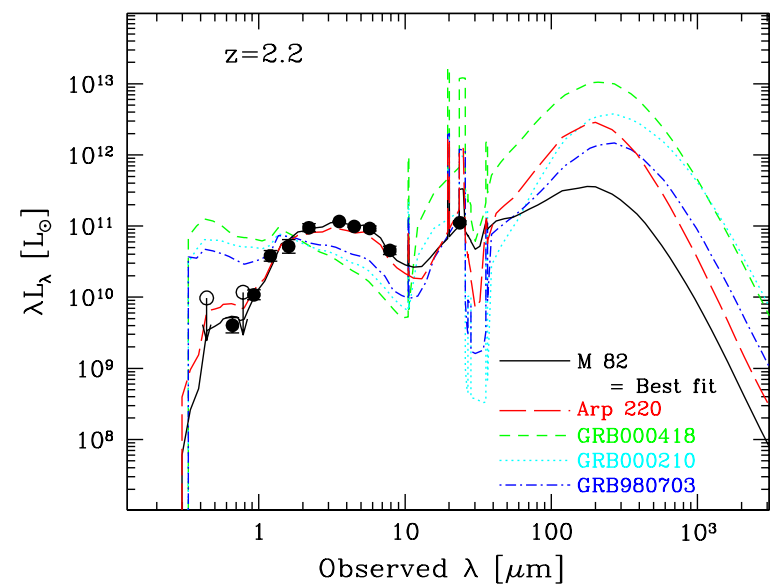

Fig. 2.- The observed SED (corrected for Galactic extinction as described in the text) of the host galaxy of GRB 080207 plotted against observed wavelength.

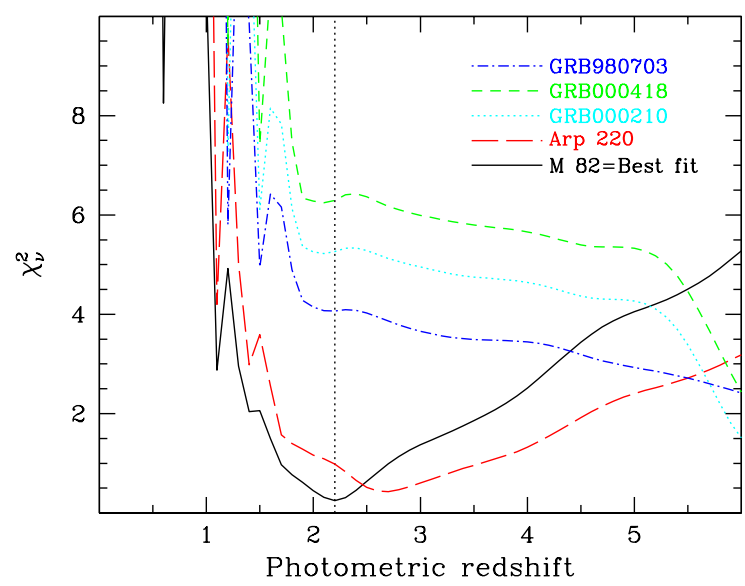

Fig. 3.- GRB 080207 host galaxy $\chi_{v}^{2}$ of the NIR bump plotted against redshift. The best-fit redshift $z=2.2$ is indicated by a dotted line. 
well predicted by the best-fit SED. At $z=2.2$, MIPS24 falls roughly on the redshifted polycyclic aromatic hydrocarbon $(\mathrm{PAH})$ feature, ubiquitous in metal-rich starbursts. The faint optical fluxes are clearly subject to significant extinction; the GRASIL starburst models predict $A_{V} \sim 1$ mag. However, this could be a lower limit since these opacities are integrated over the entire galaxy in the models, and consider the opacity of the molecular clouds, together with their filling factor over the galaxy disk.

\section{Discussion and conclusions}

We can use the GRASIL templates to infer the total infrared (IR) luminosity $\left(L_{\mathrm{IR}}\right)$ the star-formation rate (SFR), and the stellar mass $\left(M_{*}\right)$. From the SED fitting procedure, we obtain a total $L_{\mathrm{IR}} \sim 6.8 \times 10^{11} \pm 25 \% L_{\odot}$, and SFR $\sim 119 \pm 25 \% M_{\odot} \mathrm{yr}^{-1}$. This is roughly an order of magnitude lower than the SFRs implied by the few detections of GRB hosts in the submillimeter (Tanvir et al. 2004), but would definitely qualify the host of GRB 080207 as a dusty luminous star-forming galaxy.

In the absence of extinction, the inferred SFR is sufficiently high that both [O $\left.\mathrm{O}_{\mathrm{II}}\right]$ and $\mathrm{H} \alpha$ would have been detected at $>5 \sigma$ with the SINFONI spectra. The (conservative) $5 \sigma$ upper limits we obtain (in $J$ for [O II] and $K$ for $\mathrm{H} \alpha$ ) give a SFR $30-40 M_{\odot} \mathrm{yr}^{-1}$ (using the SFR calibrations of Savaglio et al. 2009), taking into account the uncertainty in redshift, and the impact of the sky lines on the noise in the observed spectrum. This is a factor of 3 to 4 times lower than the unattenuated SFR inferred from $L_{\mathrm{IR}}$ of the SED analysis; if the latter value is the true one, the extinction of the emission lines would be $A_{V} \gtrsim 1-2 \mathrm{mag}$, consistent with the SED fitting.

There is some indication that the extinction could be even higher around the GRB 080207 location. Assuming there is a spectral break between the optical and X-ray band due to electron cooling $\left(v_{C}\right)$, the optical spectral slope soon after the trigger will be $\beta_{\text {opt }}=\beta_{X}-0.5$, and the $\mathrm{R}$ magnitude is fainter for higher values of the frequency break. If $v_{C}$ coincides with the lower boundary of the XRT spectral range $(0.3$ $\mathrm{keV}$ ), the extrapolated $R$ band magnitude is $\sim 15.4,5.1$ magnitudes brighter than the photometric upper limit $\left(R_{\mathrm{AB}}>20.5\right)$. The optical flux deficit is thus significant and, if due to dust extinction, it supports independently the results we have obtained from the analysis of the galaxy SED.
The implied luminosity and SFR places the host galaxy of GRB 080207 into a subgroup of GRB host galaxies characterized by violent star formation and clearly distinct from the majority of the GRB host galaxy population (c.f., Svensson et al. 2010). A representative example is the host of GRB 010222, which is supposed to have $L_{\text {bol }} \approx 4 \times 10^{12} L_{\odot}$ and a SFR of about $600 M_{\odot} \mathrm{yr}^{-1}$ (Frail et al. 2002). Also the submm-detected hosts of GRBs 980329, 980703, 000210 and 000418 belong to this category (Berger et al. 2003; Michałowski et al. 2008), while the host of the first dark burst, GRB 970828, only has a comparatively modest SFR of $24_{-14}^{+43} M_{\odot} \mathrm{yr}^{-1}$ (Le Floc'h et al. 2006).

The stellar mass, $M_{*}$, of the host of GRB 080207 inferred from the SED fitting is $\sim 3.2 \times 10^{11} \pm 25 \% M_{\odot}$, one to two orders of magnitude higher than those found for GRB hosts at $z \lesssim 1.5$ (e.g., Savaglio et al. 2009). The SED fit gives an absolute rest-frame $K$ magnitude of the GRB 080207 host: $M_{K}(\mathrm{AB}) \sim$ -25.2. From the correlation between $M_{*}$ and $M_{K}$ derived by Savaglio et al. (2009), we infer $M_{*} \sim$ $4.0 \times 10^{11} M_{\odot}$, consistent within the uncertainties of the value derived from the SED analysis.

Figure 4 shows stellar masses plotted against redshift for GRB host galaxies and a set of GOODS galaxies (taken from Savaglio et al. 2009). As can be seen in the figure, the mass of the host of GRB 080207 is comparable to the most massive GRB host galaxies ever measured (GRBs 020127, 080325: Berger et al. 2007; Hashimoto et al. 2010). Besides being quite massive, the hosts of both GRB 020127 and 080325 are also EROSs (Berger et al. 2007; Hashimoto et al. 2010), similar to the host of GRB 080207 . The SFR and stellar mass of the GRB 080207 host are consistent with the correlation found for star-forming $\mathrm{Bz}$ selected galaxies at $z=2$ discussed by Daddi et al. (2007). The specific SFR, SSFR, of the GRB 080207 host is comparable to other galaxy populations at $z=$ 2, with SSFR $\sim 0.4 \mathrm{Gyr}^{-1}$.

The metal abundance implied from the massmetallicity relation at $z=2$ (Erb et al. 2006; Hayashi et al. 2009) places the massive host of GRB 080207 at roughly solar metal abundance. This galaxy is more chemically evolved than typical GRB hosts which (at least at lower redshifts) tend to be metal poor and much less massive (Savaglio et al. 2009). Moreover, the GRB 080207 galaxy seems to be older than many GRB hosts. The mass of the stars in the burst is $\lesssim 3 \%$ of the total stellar mass in the galaxy. This is consis- 


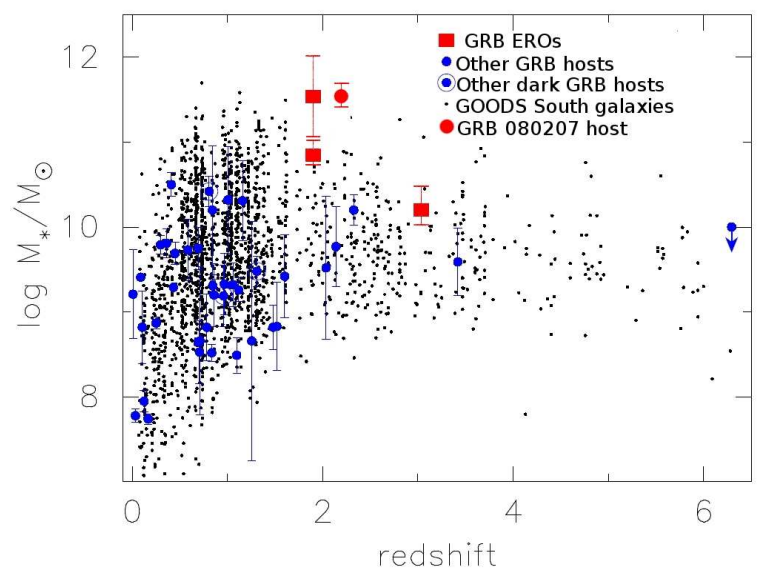

Fig. 4.- Stellar mass vs. redshift for GRB hosts and other galaxy populations. Data are taken from Savaglio et al. (2009).

tent with the clear presence of the NIR bump in the SED, and implies that the mean stellar age is relatively old, 1-2 Gyr, unlike many GRB hosts which tend to be young, $\lesssim 10 \mathrm{Myr}$ (e.g., Michałowski et al. 2008; Levesque et al. 2010).

With its extremely red $R-K$ color, $(R-K)_{\text {Vega }} \sim 6.3$, the host of GRB 080207 is the most extreme ERO found to date for a GRB host galaxy ${ }^{3}$. Moreover, the unusually high ratio of $24 \mu \mathrm{m}$-to- $R$-band flux of the GRB 080207 host makes it also a dust-obscured galaxy, or DOG (Dey et al. 2008). To our knowledge, this is the first GRB host galaxy to be so classified. Such red host colors at $z \gtrsim 2$ stand out from the typical sub-luminous blue galaxies that tend to dominate lower- $z$ GRB host populations (Savaglio et al. 2009). In fact the colors and other properties of the host of GRB 080207 are more typical of dusty $z=2$ galaxies detected in the IR and submillimeter (Pope et al. 2008), star-forming $B z K$-selected galaxies (e.g., Daddi et al. 2005; Grazian et al. 2007), or Distant Red Galaxies (e.g., van Dokkum et al. 2004). Indeed, there is more and more evidence (e.g., GRBs 020127, 080325, 080607: Berger et al. 2007; Hashimoto et al. 2010; Chen et al. 2010) that some dark bursts, those with extremely red optical-IR colors, may point to a distinct GRB host population: one that harbors more evolved, more metal enriched stellar

${ }^{3}$ The host of GRB 020127 has $(R-K)_{\text {Vega }} \sim 6.2$ (at $z=1.9$ : Berger et al. 2007), and the host of GRB $030115(R-K)_{\text {Vega }}=6$ $(z=2.6$ : Levan et al. 2006). populations, but with high SFRs and significant dust extinction.

G.C., L.K.H., and E.P. gratefully acknowledge a financial contribution from the agreement ASI-INAF I/009/10/0. A.R. and S.K. acknowledge support by DFG Kl 766/11-3, Kl 766/16-1 and by the German Academic Exchange Service (Deutscher Akademischer Austausch-Dienst; project D/08/15024). A.R. acknowledges support from the BLANCEFLOR Boncompagni - Ludovisi, née Bildt foundation.

Facilities: VLT, Spitzer, LBT, HST, Gemini.

\section{REFERENCES}

Andreev, M., Kurenya, A., \& Pozanenko, A. 2008, GCN Circular, \#7333

Berger, E., Cowie, L. L., Kulkarni, S. R., Frail, D. A., Aussel, H., \& Barger, A. J. 2003, ApJ, 588, 99

Berger, E., Fox, D. B., Kulkarni, S. R., Frail, D. A., \& Djorgovski, S. G. 2007, ApJ, 660, 504

Cenko, S. B., et al. 2009, ApJ, 693, 1484

Chen, H.-W., et al. 2010, ApJ, 723, L218

Daddi, E., et al. 2005, ApJ, 631, L13

Daddi, E., et al. 2007, ApJ, 670, 156

Dey, A., et al. 2008, ApJ, 677, 943

Elston, R., Rieke, G. H., \& Rieke, M. J. 1988, ApJ, 331, L77

Erb, D. K., Shapley, A. E., Pettini, M., Steidel, C. C., Reddy, N. A., \& Adelberger, K. L. 2006, ApJ, 644, 813

Evans, I. N., et al. 2010, ApJS, 189, 37

Fazio, G. G., et al. 2004, ApJS, 154, 10

Frail, D. A., et al. 2002, ApJ, 565, 829

Fruchter, A. S., \& Hook, R. N. 2002, PASP, 114, 144

Grazian, A., et al. 2007, A\&A, 465, 393

Greiner, J., et al. 2011, A\&A, 526, A30

Hashimoto, T., et al. 2010, ApJ, 719, 378

Hayashi, M., et al. 2009, ApJ, 691, 140 
Holland, S. T., et al. 2010, ApJ, 717, 223

Iglesias-Páramo, J., et al. 2007, ApJ, 670, 279

Jakobsson, P., Hjorth, J., Fynbo, J. P. U., Watson, D., Pedersen, K., Björnsson, G., \& Gorosabel, J. 2004, ApJ, 617, L21

Le Floc'h, E., et al. 2003, A\&A, 400, 499

Le Floc'h, E., Charmandaris, V., Forrest, W. J., Mirabel, I. F., Armus, L., \& Devost, D. 2006, ApJ, 642, 636

Levan, A., et al. 2006, ApJ, 647, 471

Levesque, E. M., Berger, E., Kewley, L. J., \& Bagley, M. M. 2010, AJ, 139, 694

Madau, P. 1995, ApJ, 441, 18

Michałowski, M. J., Hjorth, J., Castro Cerón, J. M., \& Watson, D. 2008, ApJ, 672, 817

Michałowski, M. J., et al. 2009, ApJ, 693, 347

Michałowski, M., Hjorth, J., \& Watson, D. 2010, A\&A, 514, 67

Makovoz D., \& Marleau F. R. 2005, PASP, 117, 1113

Perley, D. A., et al. 2009, AJ, 138, 1690

Pope, A., et al. 2008, ApJ, 689, 127

Racusin, J.L. et al. 2008a, GCN Circular \#7264

Racusin, J.L. et al. 2008a, GCN Circular \#7266

Racusin, J.L. et al. 2008b, GCN Report \#113.1

Rieke, G. H., et al. 2004, ApJS, 154, 25

Rol, E., et al. 2007, ApJ, 669, 1098

Rol, E., Wijers, R. A. M. J., Kouveliotou, C., Kaper, L., \& Kaneko, Y. 2005, ApJ, 624, 868

Rossi, A., et al. 2011, A\&A, submitted

Savaglio, S., Glazebrook, K., \& Le Borgne, D. 2009, ApJ, 691, 182

Sawicki, M. 2002, AJ, 124, 3050

Schlegel, D. J., Finkbeiner, D. P., \& Davis, M. 1998, ApJ, 500, 525
Silva, L., Granato, G. L., Bressan, A., \& Danese, L. 1998, ApJ, 509, 103

Simpson, C., \& Eisenhardt, P. 1999, PASP, 111, 691

Stetson, P.B. 1987, PASP, 99, 191

Svensson, K. M., Levan, A. J., Tanvir, N. R., Fruchter, A. S., \& Strolger, L.-G. 2010, MNRAS, 405, 57

Tanvir, N. R., et al. 2004, MNRAS, 352, 1073

van der Horst, A. J., Kouveliotou, C., Gehrels, N., Rol, E., Wijers, R. A. M. J., Cannizzo, J. K., Racusin, J., \& Burrows, D. N. 2009, ApJ, 699, 1087

van Dokkum, P. G., et al. 2004, ApJ, 611, 703

This 2-column preprint was prepared with the AAS ${ }^{\mathrm{AT}} \mathrm{E}_{\mathrm{E}} \mathrm{X}$ macros v5.2. 